\title{
The global distribution of the M1 ocean tide
}

\author{
Philip L. Woodworth \\ National Oceanography Centre, Joseph Proudman Building, 6 Brownlow Street, Liverpool, L3 5DA, UK
}

Correspondence: Philip L. Woodworth (plw@ noc.ac.uk)

Received: 18 December 2018 - Discussion started: 8 January 2019

Revised: 20 March 2019 - Accepted: 21 March 2019 - Published: 16 April 2019

\begin{abstract}
The worldwide distribution of the small degree$3 \mathrm{M} 1$ ocean tide is investigated using a quasi-global data set of over 800 tide gauge records and a global tide model. M1 is confirmed to have a geographical variation in the Atlantic consistent with the suggestion of Platzman (1984b) and Cartwright (1975) that M1 is generated in the ocean as a consequence of the spatial and temporal overlap of M1 in the tidal potential and one (or at least a small number of) diurnal ocean normal mode(s). As a consequence, it is particularly strong around the UK and on North Sea coasts (amplitudes $\sim 10 \mathrm{~mm}$ ). This analysis shows that their suggestion is also consistent to a great extent with the observed small amplitudes in the Pacific and Indian oceans. However, there are differences at the regional and local level which require much further study via more sophisticated ocean tidal modelling. By contrast, what is called the M1' tide (a combination of several degree-2 lines in the tidal potential with frequencies close to that of M1) is shown to have a geographical distribution consistent with expectations from other degree-2 diurnal tides, apart from locations such as around the UK where tidal interactions introduce complications. As far as I know, this is the first time that these small tidal constituents have been mapped on a global basis and, in particular, the first time that the ocean response to the degree- 3 component of the tidal potential has been investigated globally.
\end{abstract}

\section{Introduction}

M1 is a small tidal constituent with a frequency of 1 cycle per lunar day. It arises from the degree- 3 component of the tidal potential, unlike larger constituents such as M2, the predominant semidiurnal tide in the ocean with a frequency double that of M1, which originate from the degree-2 component. Agnew (2007), Pugh and Woodworth (2014) and other texts can be consulted for explanations of why the tidal potential contains degree- 2 and degree- 3 (and degree-4, etc.) components.

M1 was not identified unambiguously in tide gauge records until as late as 1968 when it was observed in data from Cuxhaven, Germany (Cartwright, 1975). Instead, what analysts referred to as "M1" was more likely to be due to other tidal lines within the "M1 group", which have frequencies slightly different from M1 itself and which originate from the degree- 2 component of the tidal potential. Cartwright $(1975,1976)$ used very long tide gauge records (several with 18 or more years of data) to demonstrate that the true degree-3 M1 was particularly large (amplitude $1 \mathrm{~cm}$ ) around the UK and on North Sea coasts. This was consistent with the suggestion of Platzman (published later in Platzman, 1984b) that M1 is forced by one (or a small number of) normal mode(s), especially one with a period of $25.7 \mathrm{~h}$ which is particularly strong in the Atlantic rather than in the other ocean basins. Amin (1982) used an additional seven long records from the west coast of Great Britain, confirming the large $\mathrm{M} 1$ in this region and showing that amplitudes increased going north.

Some years later, Cartwright et al. (1988) used 13 long tide gauge records from the North and South Atlantic, demonstrating consistency with Platzman's suggestion over a wider area. Figure 1 is copied from that paper. It shows the amplitudes and Greenwich phase lags for M1 at the 13 Atlantic locations, some of which were taken from the earlier papers (Cartwright, 1975, 1976). The co-range and co-tidal lines indicate the amplitudes and phase lags that Platzman obtained in his synthesis of M1 from about 10 normal modes of the world ocean (Platzman, 1984b), explaining, qualitatively at least, the larger amplitudes in the NE Atlantic. As Ray (2001) explained in his Fig. 2, M1 in the tidal potential is distributed symmetrically north and south of the Equator, 


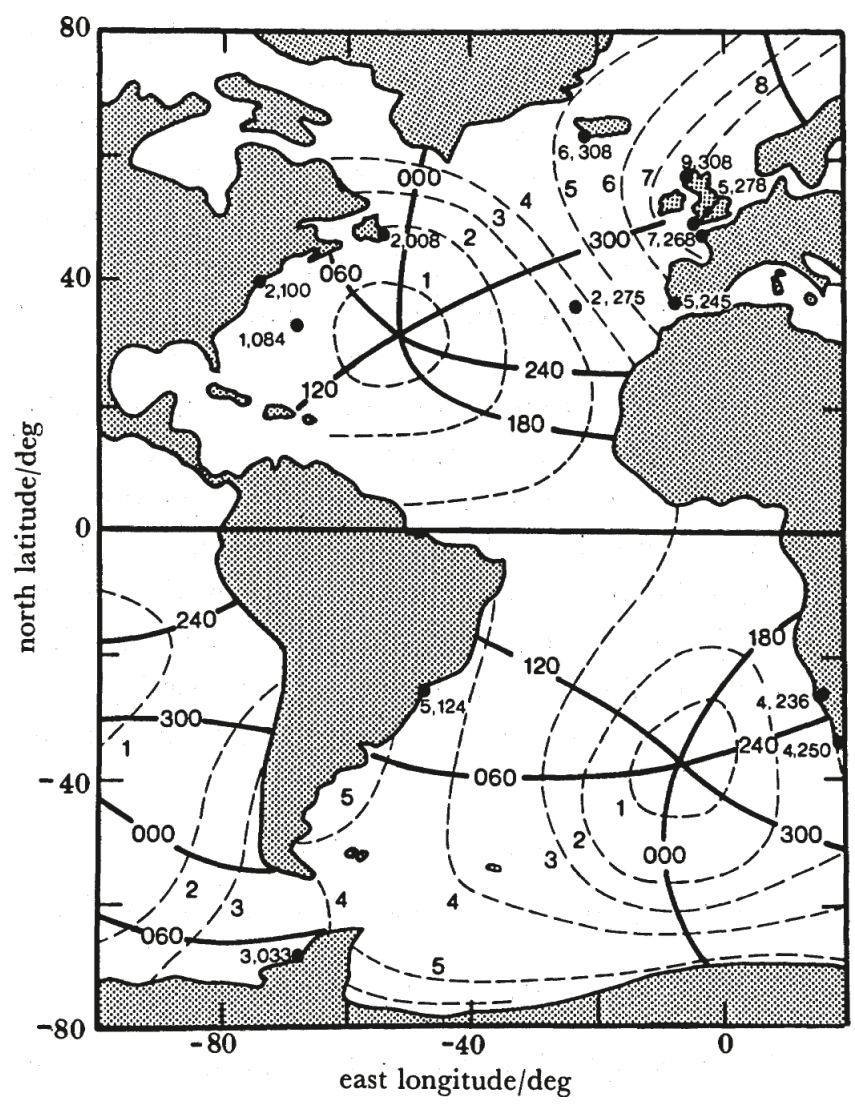

Figure 1. Contours of equal amplitude (millimetres, dashed lines), and equal phase lag (degrees, full lines) synthesized for the component M1, transposed from Fig. 9 of Platzman (1984b) with $60^{\circ}$ arbitrarily added to phase values. Black circles are positions of multiyear coastal stations at which M1 has been directly evaluated, with amplitude and phase lag shown alongside each station location. From Cartwright et al. (1988).

unlike the more familiar degree-2 diurnal potential, which is antisymmetric. The $25.7 \mathrm{~h}$ Platzman mode, the most important in the synthesis, is also largely symmetric. Consequently, the similarity of M1 in the potential with the normal mode, both spatially and temporally, leads to its preferential excitation in the Atlantic where the mode is relatively strong.

The co-range and co-tidal lines in Fig. 1 were copied from a figure in Platzman (1984b) which covered the whole ocean; that figure showing his synthesis of M1 is reproduced here as Fig. 2. One can see that, for the Platzman-Cartwright theory of the generation of M1 to be accepted as largely correct, it remains to be verified by, for example, observations of low amplitudes in the central Indian and Pacific oceans, larger amplitudes in the North Pacific, and phase differences along the American Pacific coast.

Cartwright et al. (1988) (of which the present author was a co-author) limited their study to the Atlantic and did not take the obvious next step of checking that M1 was indeed much smaller in other ocean basins, and they made the rather rash

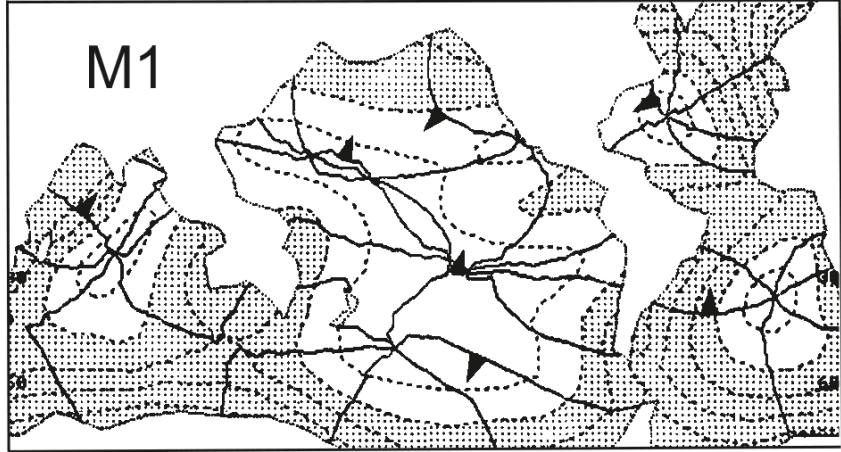

Figure 2. A map of the synthesized principal diurnal tide of third degree (M1). Amplitudes (dashed lines) are contoured at $1 \mathrm{~mm}$ intervals, and are shaded where the amplitude exceeds $2 \mathrm{~mm}$. Phases are shown at $60^{\circ}$ intervals, and the direction of phase propagation (increasing phase) is indicated by an arrowhead on contours of zero phase. This figure is copied from a panel in Fig. 9 of Platzman (1984b). Cartwright et al. (1988) had to add $60^{\circ}$ to the phases shown here in order to adequately represent the tide gauge measurements of M1 phase, as shown for the Atlantic in Fig. 1; the reasons for the offset were not understood.

statement that "We are unlikely to get any further data for this esoteric spectral line". Therefore, the aim of the present paper is to make that long-overdue extension to other ocean basins and to densify Cartwright's findings for the Atlantic, by using data from over 800 tide gauge records distributed around the world.

\section{Tidal details}

\subsection{M1 and M1'}

The "M1 group" refers to a set of tidal lines with frequencies within 1 or 2 cycles $\mathrm{yr}^{-1}$ of the frequency of M1 itself (Cartwright, 1975). ${ }^{1}$ Table 1 lists the lines in the M1 group taken from the tables of Cartwright and Tayler (1971) and Cartwright and Edden (1973). These are denoted (1) to (8), with (4) being M1, itself with a frequency of 1 cycle per lunar day or half that of M2. It has two nodal sidebands (3 and 5 ) which have approximately equal amplitudes (see below). These three result from the degree- 3 component of the potential. As regards the degree- 2 terms, one can ignore (6) which is much smaller than the other four lines (1, 2, 7 and 8$)$. The combination of these four is denoted in this paper as M1'. In principle, if one has many years (ideally 18.6 but a minimum of 9; Cartwright, 1975) of good tide gauge data, then M1 and the two largest lines in M1' (2 and 7) can be identified

\footnotetext{
${ }^{1} \mathrm{~A}$ "group" in tidal terminology is a set of lines in the tidal potential that have the same first two Doodson numbers, so that different groups within the same species are separated by 1 cycle per month (Pugh and Woodworth, 2014). Cartwright (1975) was, therefore, being careful to explain what he meant by the "M1 group".
} 
Table 1. Lines in the tidal potential within the M1 group.

\begin{tabular}{llllrrrrrr}
\hline Line & \multicolumn{3}{c}{ Doodson numbers $^{\mathrm{a}}$} & Degree & $\begin{array}{r}\text { Frequency } \\
\left(\mathrm{deg} \mathrm{h}^{-1}\right)\end{array}$ & $\begin{array}{r}\text { Amplitude }^{\mathrm{b}} \\
(\mathrm{m})\end{array}$ \\
\hline$(1)$ & 1 & 0 & 0 & -1 & -1 & 0 & 2 & 14.48520 & 0.00137 \\
$(2)$ & 1 & 0 & 0 & -1 & 0 & 0 & 2 & 14.48741 & 0.00741 \\
$(3)$ & 1 & 0 & 0 & 0 & -1 & 0 & 3 & 14.48985 & 0.00059 \\
$(4)$ & 1 & 0 & 0 & 0 & 0 & 0 & 3 & 14.49205 & 0.00399 \\
$(5)$ & 1 & 0 & 0 & 0 & 1 & 0 & 3 & 14.49426 & 0.00052 \\
$(6)$ & 1 & 0 & 0 & 1 & -1 & 0 & 2 & 14.49449 & 0.00059 \\
$(7)$ & 1 & 0 & 0 & 1 & 0 & 0 & 2 & 14.49669 & 0.02062 \\
$(8)$ & 1 & 0 & 0 & 1 & 1 & 0 & 2 & 14.49890 & 0.00414
\end{tabular}

${ }^{a}$ These are the six integer multipliers that define the angular speed of each line. In Doodson's own notation, there would be 5's added to the second-sixth numbers in order to avoid negative values and so be given as 155445 , etc. (Pugh and Woodworth, 2014). ${ }^{\mathrm{b}}$ Amplitudes in the tidal potential (normalized by acceleration due to gravity) are from the column in the tables of Cartwright and Tayler (1971) and Cartwright and Edden (1973), which refer to the mid-20th century with their signs omitted (the signs provide phase information). For degree-2, only lines with amplitudes larger than $0.00015 \mathrm{~m}$ are considered. If an amplitude is required for a particular location, then it has to be multiplied by the appropriate spatial dependence given in Table 2 of Cartwright and Tayler (1971). Note that line (4) in this list is the real M1.

individually, as in the Cartwright papers and Amin (1982). ${ }^{2}$ However, they are not separable if one has only, say, one year of data. Consequently, the energy in a tidal spectrum obtained from one year of data might have a peak around the M1 frequency, but it will not be immediately apparent whether it stems from M1 itself or from M1'.

For example, Fig. 3a shows the diurnal section of a spectrum of one year of data from Honolulu, Hawaii. The main degree-2 tidal constituents (especially $\mathrm{O} 1$ and K1) stand out clearly, as does a peak around M1. In fact, it will be shown below that this peak is almost entirely due to degree-2 M1'. A similar situation applies in Fig. 3b for Atlantic City (US Atlantic coast). Figure $3 \mathrm{c}$ has a spectrum for Cuxhaven (German North Sea coast), where M1 was first identified, and Fig. 3d has one for Newlyn (SW England). It will be seen (as in Cartwright, 1975) that most of the peaks in (c, d) are due to degree-3 M1. The important point to make is that M1 (or M1', to be decided) can usually be identified in such spectra above the non-tidal background as long as it has an amplitude of several millimetres or more.

\subsection{Usual tidal analysis procedure for M1}

In the software used at the National Oceanography Centre (NOC, Bell et al., 1998), and probably at other centres, the

\footnotetext{
${ }^{2}$ The analyses of Cartwright $(1975,1976)$, Cartwright et al. (1988) and Amin (1982) involved spectral analyses of long tide gauge records in which lines (2), (4) and (7) in Table 1 were separable. However, one must note that the notation used was different. Cartwright refers to the principal degree-2 term (i.e. line 7) as M1', whereas Amin (1982) refers to line (2) as M1' and line (7) as M1". IHO (2006) denotes lines 2 and 7 as M1B and M1A respectively. This confusing situation is not helped by misprints at the top of $\mathrm{p}$. 278 of Cartwright (1975) in which M1 and M1' are interchanged and in the header of Table 4b of Amin (1982). As explained above, the present paper takes it lead from Doodson (1928) and Doodson and Warburg (1941) to denote the combination of the four largest degree-2 terms (1, 2, 7 and 8) as M1'.
}

tidal analysis of a tide gauge record makes allowance for a constituent named M1 with a frequency of 1 cycle per lunar day. However, in spite of its name, an assumption is made that any energy at that frequency is entirely due to what I instead call M1', being a combination of the four largest degree- 2 terms $(1,2,7$ and 8$)$ in Table 1 , in the same proportion as in the tidal potential. That energy is represented as a harmonic amplitude $(H)$ and phase lag $(G)$, which are adjusted by "nodal factors" $f$ and $u$ that are time-dependent functions of the longitude of the lunar ascending node $(N)$ and the longitude of lunar perigee $(p)$. The usual expression "nodal factor" is clearly a misnomer when, as in this case of M1', the factors vary over a combination of both nodal and perigean cycles. Doodson (1928) and Doodson and Warburg (1941) show that the nodal factors for M1' can be calculated from

$f \cos u=2 \cos (p)+0.4 \cos (p-N)$,

$f \sin u=\sin (p)+0.2 \sin (p-N)$,

with a normalization in $f$ that stems from much earlier work by Darwin (see Doodson, 1921, 1928). As a consequence, its average value is approximately 1.57 and not 1.0 . It is important to keep this normalization in mind when comparisons are made to other reported amplitudes.

These expressions for $f$ and $u$ are still used in the NOC software and are shown by the black lines in Fig. 4. It can be seen that both factors change rapidly, primarily over the perigean cycle. A more exact computation, using the amplitudes in Cartwright and Tayler (1971) of the four largest degree- 2 terms, results in the red and green lines. There is little difference between the black and coloured lines, so I have continued to use Doodson's expressions for $f$ and $u$ in Eq. (1) throughout the present investigation.

\section{Data and methods}

The method used for this investigation is different to that used in Cartwright $(1975,1976)$ and Cartwright et al. (1988). Those studies involved the use of a small number of long tide gauge records (several with 18 or more years of data), from which spectral analysis allowed the separation of M1 from the two main degree- 2 lines. That method is admittedly more rigorous than the one described below, if one has long continuous records with few gaps. However, the method presented here works well for many disparate records insofar as the degree-3 M1 constituent is concerned. And in the case of degree-2 M1', it provides findings that are consistent for most of the world with Doodson's assumption that M1' is composed of the four largest degree-2 terms in proportions given in the tidal potential and consequently with the nodal factors for M1' as in Eq. (1).

The method employs records from the Global Extreme Sea Level Analysis Version 2 (GESLA-2) data set (Woodworth et al., 2017). A tidal analysis using the NOC soft- 


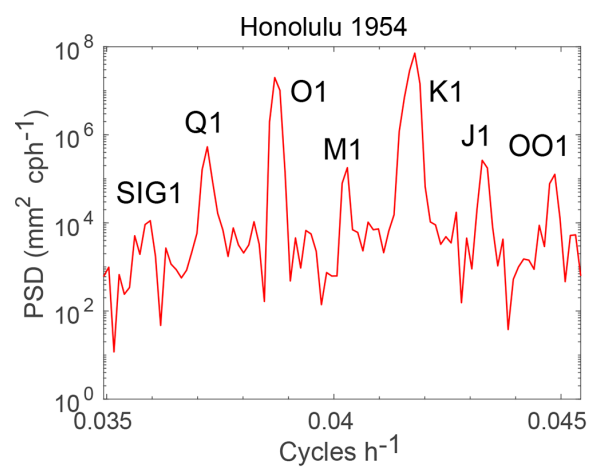

(a)

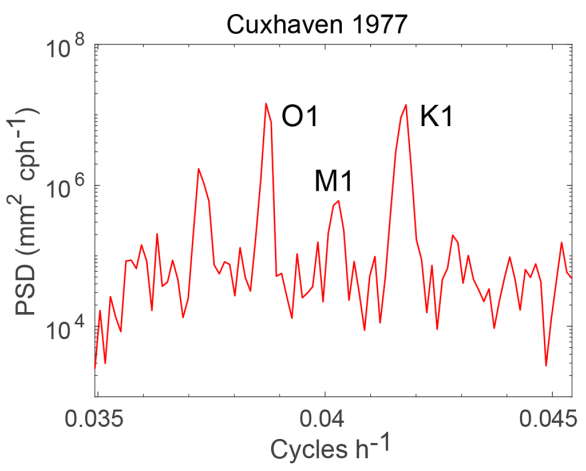

(c)

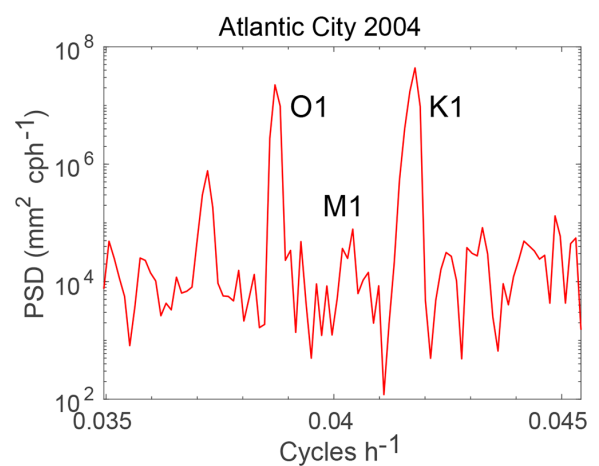

(b)

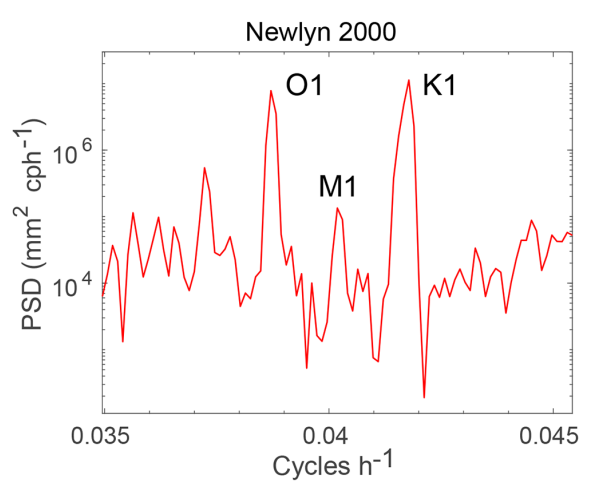

(d)

Figure 3. (a) Power spectral density (PSD) within the diurnal section of a spectrum of one year of sea level data from Honolulu, Hawaii showing the lines of the main diurnal tides. (b, c, d) The corresponding spectra from Atlantic City, Cuxhaven and Newlyn respectively.

ware was performed for each station year of data that was at least $75 \%$ complete, using a standard set of 63 constituents including M1. That provided estimates of amplitude $(H)$ and phase lag $(G)$ for that year, as explained in Sect. 2.1. Subsequently, records were required to have at least nine near-complete years of data for 1920-onwards, resulting in 804 records for study distributed around the world coastline. These come from 536 stations, there being sometimes alternative station records from more than one provider. Only 10 of these records were rejected, including those from Wilmington, North Carolina, at which tides are known to have been affected by river dredging (e.g. Ray, 2009) and several which had anomalous phase lags of the main tidal constituents, probably due to tide gauge timing errors.

The next step is to un-correct the estimated $H$ and $G$ for the nodal adjustments of Eq. (1) that had been made in the tidal software. In other words, one calculates $h=H f$ and $\varphi=G-u$, where the instantaneous (mid-year) amplitude $(h)$ and phase $(\varphi)$ can be thought of as representing an M1 carrier wave, which at this stage could be due to either degree-3 M1 or degree-2 M1' or a combination of the two. One can then parameterize $h$ and $\varphi$ obtained for each year as a combination of the contributions from M1', which is the only part of what the software calls M1 that genuinely has the nodal variations of Eq. (1), and M1 plus its nodal sidebands (3, 4 and 5 in Table 1). This parameterization takes the form

$$
\begin{aligned}
& h \cos (\varphi)=H_{2} f \cos \left(G_{2}-u\right)+H_{3} F \sin \left(G_{3}-U\right), \\
& h \sin (\varphi)=H_{2} f \sin \left(G_{2}-u\right)-H_{3} F \cos \left(G_{3}-U\right),
\end{aligned}
$$

where the nodal factors for M1 ( $F$ and $U$ ) are approximately $F=1.0-0.28 \cos (N)$ and $U=0.0$. The derivation of $F$ and $U$ for M1 with its approximately equal nodal sidebands is similar to that for $\mathrm{Mm}$ in Appendix A of Woodworth and Hibbert (2018). The nodal factors for M1' $(f$ and $u)$ are the Doodson ones described in the previous section.

The mixture of sines and cosines in Eqs. (2a) and (2b), and their signs, originate from the way these constituents are parameterized in the harmonic expansion employed in the tidal analysis. The use of Eq. (2) assumes that $f$ and $u$ in reality are close to their values in Eq. (1) and that $F$ and $U$ also have their equilibrium form. A least-squares search is then made for the choice of $H_{2}$ and $G_{2}$ (the amplitude and phase lag of M1') and $H_{3}$ and $G_{3}$ (the amplitude and phase lag of M1) that best describes Eq. (2a) and (2b) for all the 


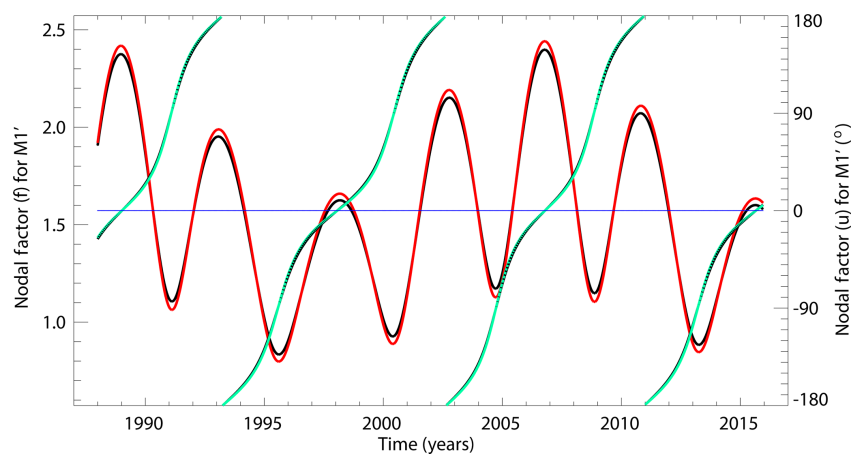

Figure 4. Variation of $f$ as given by Eqs. (1a) and (1b) as a function of time (in black), with an average value of approximately 1.57 owing to an historical normalization explained in Doodson (1928). This is accompanied closely (in red) by values one would obtain using amplitudes in Cartwright and Tayler (1971), normalized so as to have the same average value. The $f$ values are with respect to the left axis. And variations in $u$ from Eqs. (1a) and (1b) (in black) are closely accompanied (in green) by the corresponding values using Cartwright and Tayler (1971). The $u$ values are with respect to the right axis. The $f$ values in the second case span a slightly larger range than in the first case, while $u$ values in the two cases are almost identical and so the black and green curves overlap.

years in the record. The fit is aided by $f$ and $u$ varying so rapidly from year to year.

The method results in reasonable agreement between M1' phase lag and those of Cartwright $(1975,1976)$ and Cartwright et al. (1988), as shown in Table 2. There is agreement to within $\sim 20^{\circ}$ except for Southend and possibly Dunkerque. However, one should note that Cartwright's M1' was the largest degree-2 term only (i.e. line 7 in Table 1), whereas in the present analysis M1' refers to the combination of four terms, so comparison will not be perfect. In addition, because of the Doodson normalization of $f$ values mentioned above, the amplitudes for M1' from this analysis appear systematically lower than Cartwright's. Reasonable agreement can be seen in most cases at the $\sim 1 \mathrm{~mm}$ level if that is taken into account (see further below).

On the other hand, the present method results in almost identical findings for M1 to those of Cartwright $(1975,1976)$ and Cartwright et al. (1988). Results are also close to those of Amin (1982) for seven stations on the west coast of Great Britain and of Ray (2001) for three European stations (Table 3).

The Supplement provides a list of the M1' and M1 values obtained at all locations in the present work. For example, with reference to Fig. 3, M1' at Honolulu can be seen from the list to have an amplitude of $4.8 \mathrm{~mm}$, whereas that of $\mathrm{M} 1$ is only $0.8 \mathrm{~mm}$. At Atlantic City, M1' and M1 have amplitudes in a similar proportion to Honolulu. On the other hand, M1 dominates at Cuxhaven and Newlyn.

\section{Results for M1' and M1}

\subsection{Maps of M1'}

The amplitudes and phase lags for M1' are shown in Fig. 5a and $\mathrm{b}$ respectively. Amplitudes are $\sim 10 \mathrm{~mm}$ or more along the Pacific coasts of N America, SE Asia, China and Japan (but much lower on the Sea of Japan side), on the north coast of Australia, and in the NW Indian Ocean. Phase lags show spatial consistency where amplitudes are large, e.g. in the North American Pacific and around Australia.

However, it can be difficult to arrive at conclusions from an inspection of coloured dots on maps like this (especially the phase lags). Therefore, the following method is useful in demonstrating that Fig. 5 is consistent with expectations for a degree-2 diurnal tide. Figure S1a shows a modern global map for K1, the main degree-2 diurnal tide. A map of a constituent with a similar frequency (such as P1) will look much the same, although with smaller overall amplitudes and small differences in the patterns of co-range and co-tidal lines. Another diurnal tide such as $\mathrm{O} 1$, with a larger difference in frequency to that of $\mathrm{K} 1$, will have a map with much larger differences, owing to the response of the ocean to forcing being different at the two frequencies. However, as the frequency of M1' is midway between those of $\mathrm{K} 1$ and O1, its map should look something like an average of the two.

This can be tested by plotting values for $\mathrm{K} 1$ and $\mathrm{O} 1$ in the complex plane (Fig. 6a), with amplitudes normalized by their average amplitudes in the data set (alternatively their amplitudes in the tidal potential could be used). They will be at different points in the plane because of the difference in the local response to forcing. One would expect M1' to be located at a point in the plane midway between them, as shown by the open circle in Fig. 6a. That point has an amplitude $R$. The mismatch between the measured M1' (after similar normalization) and the midway point has a length $D$. Consequently, $D / R$ provides as assessment of the accuracy of measurement of M1'. Figure $6 \mathrm{~b}$ shows a histogram of this ratio, selecting stations for which $\mathrm{K} 1$ amplitude is larger than $10 \mathrm{~cm}$, thereby excluding stations located near diurnal amphidromes. It suggests that in most cases M1' has been estimated with an accuracy of about $10 \%$, at least for large M1', and it implies that the use of Eq. (1) for the nodal factors of M1' was largely correct at most places. The requirement of a minimum $\mathrm{K} 1$ amplitude inevitably means that this test is performed for the higher-amplitude regions in Fig. S1a. Supplement Fig. S2a shows a worldwide map of $D / R$ showing that it is largely a simple reflection of areas of ocean with large and small diurnal amplitudes, although with especially large values on the NW European continental shelf.

Amin $(1982,1985)$ obtained interesting results from his analysis of long records from the west coast of Great Britain, finding line (2) to be several times larger than (7), whereas one would have expected (2) to be about 3 times smaller than (7) based on the tidal potential (Table 1). These results 
Table 2. Amplitudes (mm) and Greenwich phase lags $\left(^{\circ}\right)$ for M1' and M1 as reported in Cartwright (1975, 1976) and Cartwright et al. (1988). Figure 5 of the latter shows M1 values for 13 stations, of which 6 had been reported in the earlier papers. In the right-hand columns are the corresponding values obtained in the present analysis. ${ }^{\mathrm{d}}$

\begin{tabular}{|c|c|c|c|c|c|c|c|c|}
\hline & \multicolumn{4}{|c|}{ Cartwright papers } & \multicolumn{4}{|c|}{ Present analysis } \\
\hline & \multicolumn{2}{|c|}{ M1' } & \multicolumn{2}{|c|}{ M1 } & \multicolumn{2}{|c|}{ M1' } & \multicolumn{2}{|c|}{ M1 } \\
\hline & $\mathrm{H}_{2}$ & $G_{2}$ & $H_{3}$ & $G_{3}$ & $H_{2}$ & $G_{2}$ & $H_{3}$ & $G_{3}$ \\
\hline \multicolumn{9}{|l|}{ Cartwright (1975) } \\
\hline Newlyn, Cornwall ${ }^{\mathrm{a}}$ & 1.2 & 38 & 4.8 & 278 & 1.2 & 32 & 4.6 & 274 \\
\hline Scilly Isles & 3.1 & 43 & 5.0 & 276 & 1.8 & 24 & 4.6 & 272 \\
\hline Brest, Brittany & 3.0 & 31 & 6.9 & 268 & 2.0 & 28 & 7.0 & 262 \\
\hline Malin Head, Eire & 5.6 & 101 & 8.9 & 312 & 2.0 & 72 & 9.0 & 320 \\
\hline Stornoway, Hebrides & 6.7 & 81 & 8.8 & 308 & 2.4 & 62 & 10.0 & 296 \\
\hline Lerwick, Shetland & 3.7 & 91 & 7.4 & 330 & 2.2 & 90 & 7.0 & 330 \\
\hline Southend, Essex & 2.5 & 88 & 9.5 & 112 & 2.0 & 174 & 8.4 & $116^{\mathrm{e}}$ \\
\hline Dunkerque, France & 6.6 & 101 & 9.2 & 104 & 2.0 & 154 & 8.0 & 98 \\
\hline Cuxhaven, Germany & $\mathrm{b}$ & $\mathrm{b}$ & 9.2 & 214 & 1.6 & 282 & 9.2 & 212 \\
\hline Terceira, Azores & 2.2 & 41 & 2.1 & 275 & 1.4 & 22 & 2.8 & $252^{\mathrm{f}}$ \\
\hline St. Georges, Bermuda ${ }^{c}$ & 3.4 & 191 & 1.0 & 84 & 2.6 & 188 & 0.8 & 124 \\
\hline \multicolumn{9}{|l|}{ Cartwright (1976) } \\
\hline Lagos, Portugal & 4 & 5 & 5 & 245 & 2.6 & 358 & 4.4 & 242 \\
\hline \multicolumn{9}{|l|}{ Cartwright et al. (1988) } \\
\hline Reykjavík, Iceland & & & 6 & 308 & & & 4.2 & 310 \\
\hline St. John's, Newfoundland & & & 2 & 8 & & & 3.6 & 4 \\
\hline Atlantic City, USA & & & 2 & 100 & & & 0.6 & 82 \\
\hline Cananéia, Brazil & & & 5 & 124 & & & 5.0 & 124 \\
\hline Port Nolloth, S. Africa & & & 4 & 236 & & & 3.6 & 228 \\
\hline Simons Bay, S. Africa & & & 4 & 250 & & & 4.0 & 244 \\
\hline Vernadsky, Antarctica & & & 3 & 33 & & & 3.4 & 38 \\
\hline
\end{tabular}

a Average of three separate Newlyn records reported in Cartwright (1975). ${ }^{b}$ M1' was said by Cartwright (1975) to be below noise level at Cuxhaven. ${ }^{c}$ The Bermuda M1 phase lag is given as $260^{\circ}$ in Cartwright (1975) and $84^{\circ}$ in Cartwright et al. (1988). The latter seems to be the more reliable. ${ }^{d}$ Where there was more than one alternative record for a station in the GESLA-2 data set, the records from the British Oceanographic Data Centre were used in this table for comparison to the British stations in Cartwright's papers. Similarly, records from French, German, Icelandic and US national agencies were used where available (REFMAR, BSH, Coastguard and NOAA respectively). Otherwise, records were used from the University of Hawaii Sea Level Center. See the Supplement for a full list of M1' and M1 values from the present analysis. Note also that M1' in Cartwright $(1975,1976)$ refers to line (7) in Table 1 only, while in the present analysis it refers to the combination of four lines $(1,2,7,8) .{ }^{\mathrm{e}}$ Sheerness used in the present analysis.

${ }^{\mathrm{f}}$ Ponta Delgada used in the present analysis.

would invalidate Doodson's combination of four terms into M1' and the nodal factors of Eq. (1), at least for this area of ocean. Amin's findings for these stations have recently been confirmed by analysis of very long ( $\sim 40$-year) UK records by Richard Ray, personal communication (2018). He concluded that line (7), which is the largest contributor to M1' in the potential, is suppressed in this area by a compound tide NO1, which has the same frequency as line (7) and results from the interaction between N2 and O1. Ray's analysis also presented evidence for a sizeable SO1 (interaction between $\mathrm{S} 2$ and O1), which lends credibility to the existence of NO1.

Anomalous M1' in this area in the present analysis is demonstrated by Amin's west coast stations having $D / R$ ratios in the range 0.4-1.4, as do other UK stations with the exception of SW England (Fig. S2b), even though most stations in this area have decimetric amplitudes for $\mathrm{K} 1$ and $\mathrm{O} 1$ (Pingree and Griffiths, 1982). Neighbouring stations on the European coasts have smaller values of $D / R$. Therefore, M1' in this region has a different character to M1' in other areas of ocean that also have at least decimetric diurnals but which have smaller $D / R$ ratios, as shown in Fig. $6 \mathrm{~b}$.

To some extent, this interpretation is supported by looking at the variance $(V)$ of the residuals of Eqs. (2a) and (2b), i.e. the sum of the squares of the differences between the left and right sides of Eqs. (2a) and (2b) for all $N$ years of data, divided by $N$. Figure S3a shows a histogram of $V$ for all the records in the analysis and Fig. S3b provides a worldwide map. Its median value is $0.037 \mathrm{~cm}^{2}$, corresponding to a stan- 
Table 3. Amplitudes (mm) and Greenwich phase lags $\left(^{\circ}\right)$ for $\mathrm{M} 1$ as reported in Amin (1982) and Ray (2001) and the corresponding values in the present analysis.

\begin{tabular}{|c|c|c|c|c|}
\hline & \multicolumn{2}{|c|}{ Amin (1982) } & \multicolumn{2}{|c|}{ Present analysis } \\
\hline & $H_{3}$ & $G_{3}$ & $H_{3}$ & $G_{3}$ \\
\hline Millport & 7.5 & 348 & 8.8 & 336 \\
\hline Portpatrick & 7.0 & 2 & 7.8 & 348 \\
\hline Heysham & 5.9 & 352 & 10.2 & 358 \\
\hline Liverpool & 7.2 & 30 & 9.8 & 4 \\
\hline Holyhead & 5.7 & 332 & 7.2 & 330 \\
\hline Fishguard & 5.0 & 313 & 6.2 & 310 \\
\hline \multirow[t]{3}{*}{ Milford Haven } & 5.4 & 299 & 5.8 & 288 \\
\hline & \multicolumn{2}{|c|}{ Ray (2001) } & \multicolumn{2}{|c|}{ Present analysis } \\
\hline & $H_{3}$ & $G_{3}$ & $H_{3}$ & $G_{3}$ \\
\hline Newlyn & $4.67 \pm 0.22$ & $275 \pm 3$ & 4.6 & 274 \\
\hline Lerwick & $7.25 \pm 0.18$ & $332 \pm 2$ & 7.0 & 330 \\
\hline Vigo & $5.48 \pm 0.15$ & $232 \pm 2$ & 5.2 & $230^{*}$ \\
\hline
\end{tabular}

* Using the record from the University of Hawaii in the GESLA-2 data set for the present analysis instead of the record from the Instituto Español de Oceanografia, which gives an amplitude of $5.4 \mathrm{~mm}$ and phase lag of $248^{\circ}$.
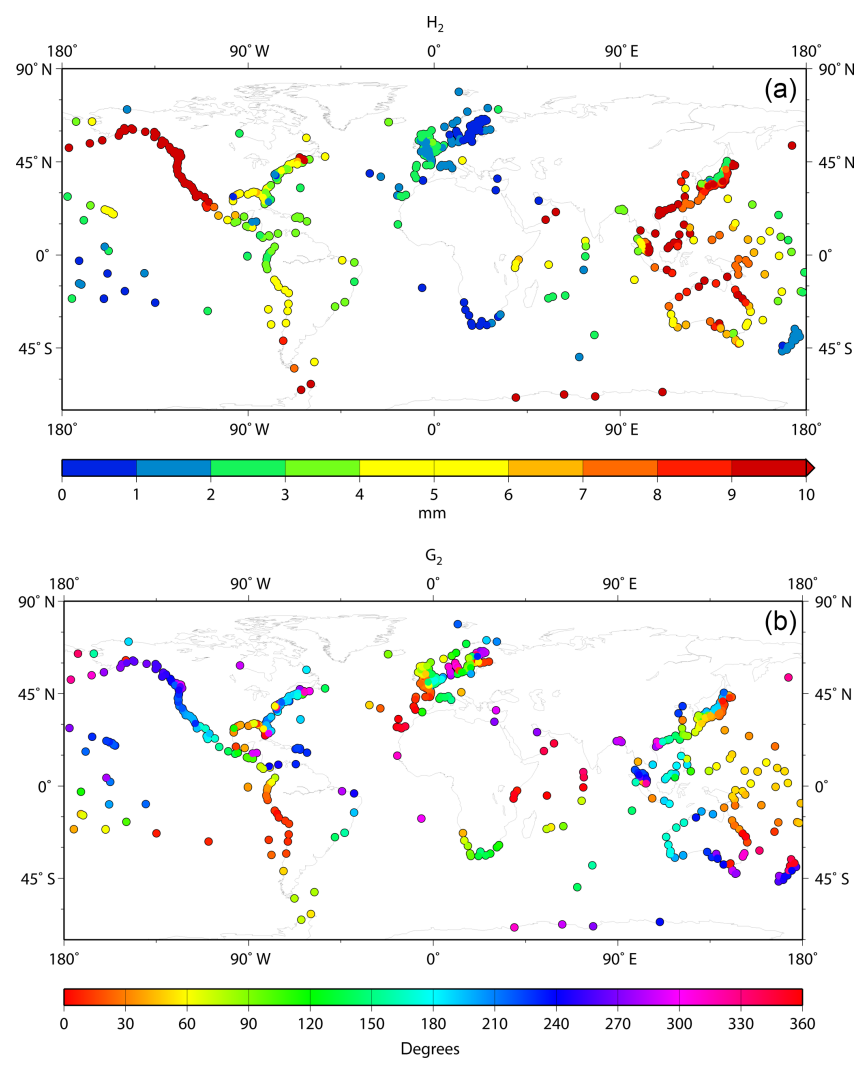

Figure 5. (a) Amplitude and (b) Greenwich phase lag of M1' at stations in the GESLA-2 data set.
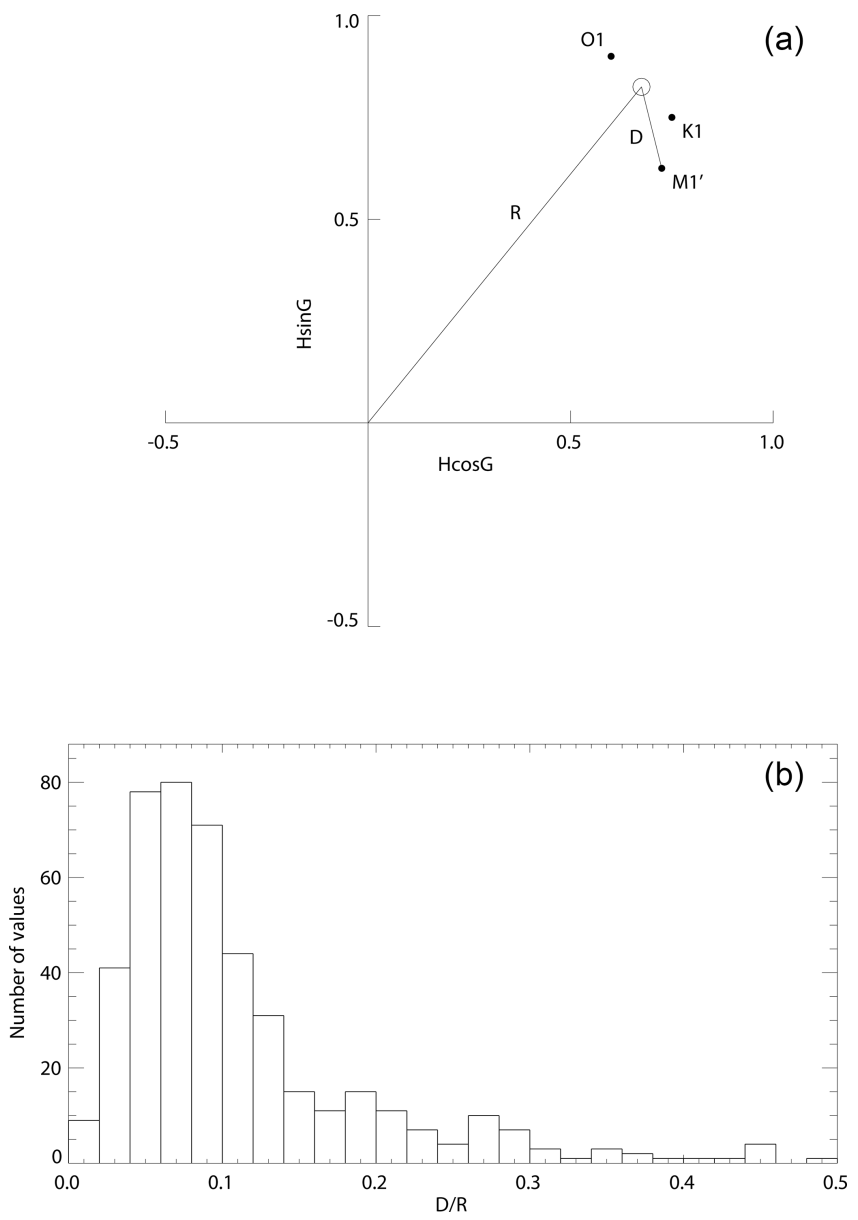

Figure 6. (a) A schematic example of the positions of $\mathrm{K} 1$ and $\mathrm{O} 1$ in the complex plane, their midway position (open circle) and its amplitude $(R)$, and the distance $(D)$ between the measured M1' and the midway point. (b) A histogram of values of the ratio $D / R$ obtained from records in GESLA-2 requiring the amplitude of $\mathrm{K} 1$ to be at least $10 \mathrm{~cm}$.

dard deviation of $2 \mathrm{~mm}$. However, around the UK there can be much larger values (Fig. S3c), especially in the shallow areas of the Irish Sea and southern North Sea. In these cases, $V$ is more typically $0.1-0.2 \mathrm{~cm}^{2}$ or a standard deviation of 3-4 mm. A large $V$ could result from a poor-quality record, or from the parameterization of Eq. (2) being inappropriate or both. It is hard to decide which explanation applies to a particular record. However, the spatial consistency shown in Fig. S3c suggests that the parameterization for M1' might be to blame in these large- $V$ areas. Lower values of $V$ are found in SW England, towards the open ocean.

Cartwright $(1975,1976)$ concentrated only on line (7), presumably because his expectations from the potential led him to believe that it would also be the most important degree2 term in UK waters. His results for M1' are in reasonable agreement with those obtained here (Table 2), with the possible exceptions of Southend and Dunkerque, as mentioned 
above. It can be seen from Fig. S3c that stations with lower values of $V$ correspond to better agreement in Table 2 and that higher values apply to the west coast of Great Britain area of Amin's stations and to the southern North Sea area of Southend and Dunkerque. Therefore, if Amin and Ray are correct that an interaction term (NO1) also plays a role at the frequency of line (7) at some locations, then Cartwright's focus on (7) and our computation of M1' in the larger- $V$ areas will not be so meaningful. Nevertheless, the findings for M1' for the UK overall (Fig. S4a, b) do appear similar to those for other diurnals (K1 and O1), having somewhat lower amplitudes on the west coast of Great Britain than the east coast and a clockwise rotation of phase lag around most of the coast (Pingree and Griffiths, 1982).

For completeness, Fig. S3d, e provide maps of $V$ for NE and NW America respectively. Spatial consistency is again demonstrated for the largest values of $V$ in the Maritime provinces of Canada, Mid-Atlantic Bight and between Vancouver Island and the mainland, all shallow-water areas where diurnal amplitudes are large and where interaction contributions to M1' are plausible. Similarly there are large values along the coasts of China and SE Asia (Fig. S3b).

\subsection{Maps of M1}

Figure 7a, b shows the corresponding maps for M1 amplitude and phase lag respectively. As shown by Cartwright (1975, 1976), Amin (1982) and Cartwright et al. (1988), there are large $(\sim 1 \mathrm{~cm})$ amplitudes around the UK and along North Sea coastlines. There are moderate (several millimetre) amplitudes in NE Canada, as Cartwright (1975) suggested might be the case, but much smaller amplitudes to the south along most of the North American Atlantic coast, until one reaches the Gulf of Mexico and the Caribbean where moderate values are obtained again. Similarly, amplitudes of several millimetres are found on the American and African coasts of the South Atlantic. Figure 7b shows M1 phase lag, confirming at a large scale the rotations in the North and South Atlantic from Platzman's synthesis discussed by Cartwright et al. (1988). The co-tidal lines from the synthesis taken from Figure 1 are also shown; they help to guide the eye around the coloured dots on the coastlines.

Amplitudes of M1 are very small for most of the central Pacific. For example, as mentioned above, the M1 amplitude at Honolulu (Fig. 3a) is only $0.8 \mathrm{~mm}$ compared to $4.8 \mathrm{~mm}$ for M1'. An exception is the coast of the Canadian Pacific and Alaska where amplitudes are $\sim 3 \mathrm{~mm}$ as they are for southern Chile. Values of several millimetre are obtained around Australia, with larger values on the west and north coasts (although at least one of the red dots in north Australia in Fig. 7a is anomalous, being from Wyndham which is up a river, while the red dot in South Australia is for Port Pirie in Spencer Gulf). Amplitudes are smaller on the east side of New Zealand than on its west coast. Moderate values $(\sim 4 \mathrm{~mm})$ are found for SE Asia and southern Africa,
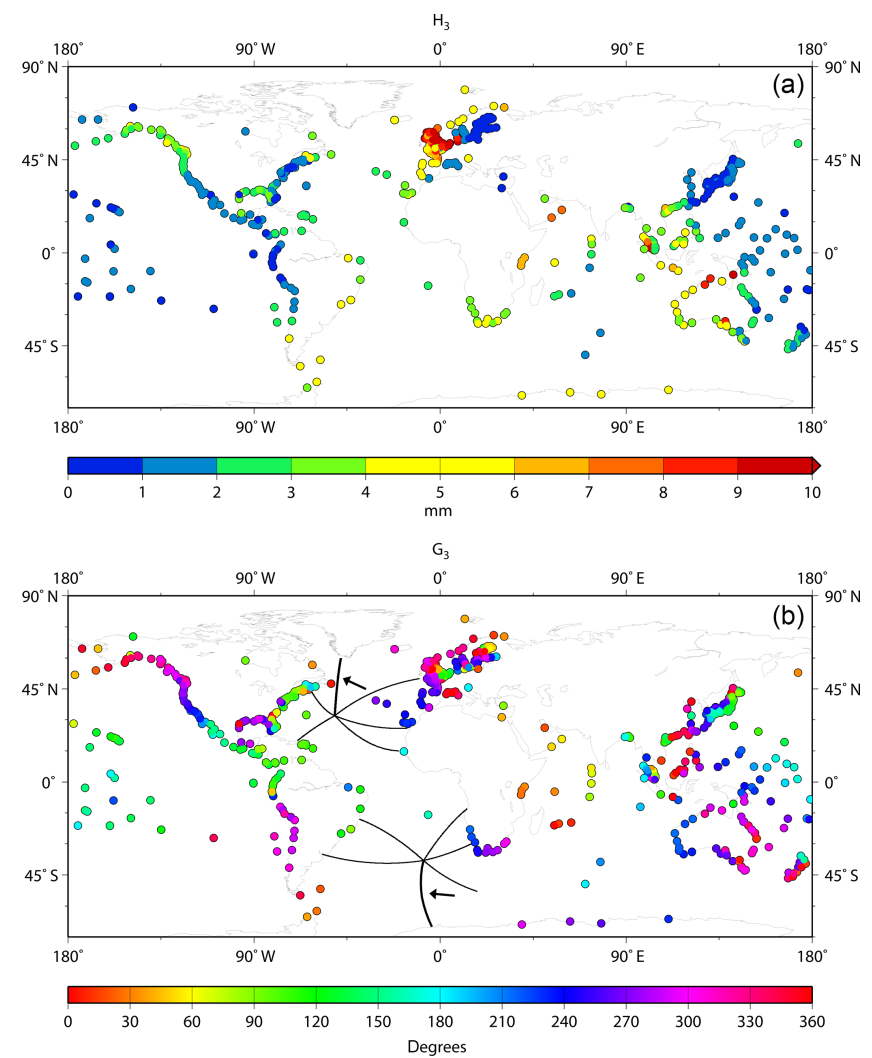

Figure 7. (a) Amplitude and (b) Greenwich phase lag of M1 at stations in the GESLA-2 data set. Panel (b) includes the co-tidal lines from the Platzman synthesis for M1 shown in Fig. 1.

while there are higher amplitudes $(\sim 7 \mathrm{~mm})$ in the NW Indian Ocean, where there are similarly high amplitudes for M1'. (A separate analysis for Karachi, Pakistan, of a combination of two short records, which are individually shorter than the 9 years required in Section 3 and so do not appear in Fig. 7, confirms a similarly high amplitude for M1 of $\sim 6.2 \mathrm{~mm}$ in the NW Indian Ocean.) Amplitudes at only the 1-2 $\mathrm{mm}$ level are observed for several islands in the central Indian Ocean. Overall, these features in Fig. 7a are qualitatively consistent with Platzman's synthesis in Fig. 2.

Figure $7 \mathrm{~b}$ shows several interesting features for M1 phase lag other than those already pointed out for the Atlantic by Cartwright et al. (1988), such as differences between the central part of the American Pacific coast and the northern and southern sections of that coastline. Contrasts in M1 phase lag can be seen in Fig. 7b between the east and west sides of New Zealand, Australia and Japan. There is reassuring consistency in both amplitude and phase information between the small number of stations in the NW Indian Ocean and similarly between stations in the Indian Ocean sector of the Antarctic coast. The clockwise phase rotation in the Indian Ocean in Fig. 2 might be supported by Fig. 7b, but there is no evidence for a similar rotation in the central Pacific. 
Additional remarks can be made on M1 from Fig. S5a-f which focus on NW Europe, NE and NW America.

As regards the UK, an increase in the amplitude of M1 can be seen travelling north on the west side of Great Britain (Fig. S5a), confirming the results of Cartwright (1975) and Amin (1982). Amplitudes are mostly smaller on the west coast of Great Britain than the east coast, although they are particularly large in the Bristol Channel (amplitudes of 24, 20 and $15 \mathrm{~mm}$ at Avonmouth, Newport and Hinkley Point respectively). Amplitudes are lower on the south coast of England than on the adjacent French coast, although the phases are similar. In addition, there is a phase difference of $\sim 180^{\circ}$ between the central-west and south-east coasts of Great Britain (Fig. S5b). Phase lag is smaller in the SW and NW than in the centre of the west coast, which is consistent with Amin (1982). He found it hard to decide on the spatial variation of phase lag around the coast from only seven stations, but the pattern is now quite clear from the many additional records. The Atlantic coast of France is $180^{\circ}$ out of phase with the southern North Sea. The phase lag in NE Scotland is similar to that along the Norwegian coast.

Large amplitudes $(\sim 10 \mathrm{~mm})$ are found at stations on the European coast of the North Sea, including Cuxhaven, with phase lag appearing to increase going north from the Netherlands to Norway. Amplitudes are lower $(\sim 5 \mathrm{~mm})$ along the Norwegian coastline. Further east, Voinov (2011) calculated amplitudes for M1 of $7 \mathrm{~mm}$ at two stations in the Barents Sea and 2 and $5 \mathrm{~mm}$ at two stations in the Kara Sea. Figure S5a shows amplitudes at the 1-2 mm level in the Baltic and Mediterranean, with the notable exception of Trieste, Italy, where amplitudes were obtained in the present analysis of $4.6 \mathrm{~mm}$ for M1' and $4.0 \mathrm{~mm}$ for M1. An M1 amplitude of $9.1 \mathrm{~mm}$ was reported at Trieste by Mosetti and Manca (1972), which was probably due to a combination of the two.

Figure S5c demonstrates how moderate amplitudes $(\sim$ $4 \mathrm{~mm}$ ) in NE Canada and in the Gulf of Mexico are separated by an extensive section of Atlantic coast where M1 amplitudes are minimal. Two stations with larger M1 amplitudes are located in the Delaware estuary, where local tidal processes are probably the cause. Even though most amplitudes in this region are very small, the phase lags obtained for most stations are consistent with those of their neighbours, providing confidence in the findings (Fig. S5d). Two exceptions are located in the Chesapeake estuary where again local processes are probably affecting the tides (Ross et al., 2017).

Amplitudes on the Pacific coast of NW America increase as one travels north of San Francisco (Fig. 7a), with values of typically $3-4 \mathrm{~mm}$ on the Canadian and Alaskan coast (Fig. S5e) and larger values of 4-6 $\mathrm{mm}$ between Vancouver Island and the mainland, where enhancements of the degree2 diurnal tides are also found (Foreman et al., 2000). Phase lags are consistent from station to station with an exception of Anchorage at the head of the Cook Inlet (Fig. S5f).

\subsection{A numerical model for M1}

The Platzman normal modes were computed many years ago on a coarse grid, with the M1 synthesis constructed from a combination of a small number of non-dissipative modes, together with a variational treatment to accommodate dissipation (Platzman, 1984a, b). Instead of following that approach in the present work, it was decided to construct a numerical model for M1 in which the modes would, in principle, be included implicitly.

The model is a global version of the regional finitedifference tide-surge model of Roger Flather (e.g. Flather, 1988; R. A. Flather, unpublished lecture notes at the International Centre for Theoretical Physics, Trieste, Italy, 1988) driven only by the tidal potential. The present version uses a 1/4-degree bathymetry derived from the General Bathymetric Chart of the Oceans (GEBCO) (Weatherall et al., 2015). It must be stressed that the model can in no way be compared to the several state-of-the-art global tide models now available, most of which make use of information from satellite altimetry (Stammer et al., 2014). Instead, it should be considered as a tool for the present purpose of investigating the spatial variation of M1. The Supplement provides details of model construction.

A first step was to obtain an optimum tuning of the model for different selections of bottom friction and horizontal eddy viscosity, each time driven only by the tidal potential for the four main degree-2 constituents only (M2, S2, K1 and O1). When acceptable global maps for M2 and K1 were obtained, then the model was considered tuned. (The Supplement contains more details on how well M2 and K1 are simulated.) A second step was to run the model using only M2, S2 and M1. In this case, $\mathrm{K} 1$ and $\mathrm{O} 1$ were left out of the forcing in order to avoid any leakage into M1 during a short (14-day) model run. In this exercise for M1, the driving potential is not proportional to $\sin (2 \varphi)$ as for degree-2 diurnals (where $\varphi$ is latitude) but has a value of $0.0013 \cos (\varphi)\left[5 \sin ^{2}(\varphi)-1\right] \mathrm{m}$ (Cartwright and Tayler, 1971). This is a tiny amplitude, which becomes even smaller when multiplied by a degree3 diminishing factor of 0.80 (Wahr, 1981). Nevertheless, it will be seen to be capable of generating an M1 of the order of $1 \mathrm{~cm}$ in parts of the ocean, such as the NE Atlantic, as discussed above.

Figure $8 \mathrm{a}, \mathrm{b}$ shows the resulting model amplitudes and phase lags for M1 using the same colour scales as for the tide gauge data in Fig. 7a, b. Figure 8a indicates larger amplitudes in the NE Atlantic, NW Australia and the NW Indian Ocean that are largely supported by the tide gauge data. Moderate amplitudes are also found in NE Canada, Gulf of Mexico, the South Atlantic coasts of America and Africa, the NE Pacific American coast, and along the Antarctic coastline. (See the Supplement for mention of large modelled amplitudes also for K1 around Antarctica.) The US Atlantic coast has very low amplitudes, as do the extensive central areas of the Pacific and Indian oceans. 


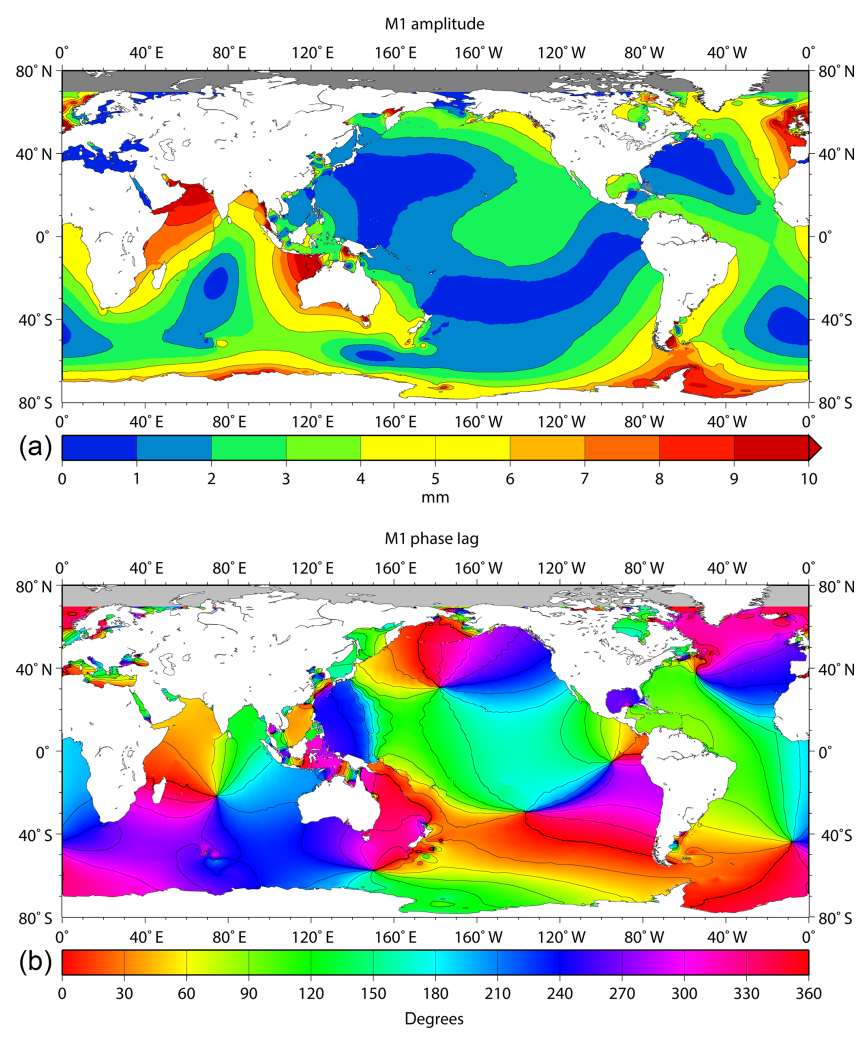

Figure 8. (a) Amplitudes and (b) Greenwich phase lags of M1 from the numerical model.

Model phase lags are shown in Fig. $8 \mathrm{~b}$ as a colour grid to enable easier comparison to those obtained from tide gauges in Fig. 7b. The two main amphidromic centres in the North and South Atlantic can be seen, although at slightly different positions than in Fig. 1. In addition, there is a clear pattern of rotation in the central Indian Ocean, different phase lags on the west and east coasts of Australia, and even similar phases in model and tide gauge data across the Pacific. The variation of phase lag along the Pacific coast of the Americas is similar to that in the tide gauges, except near the Equator where the phase varies rapidly. Overall, there can be seen to be reasonable agreement between model and data.

Many aspects of the spatial variation of M1 described by the model and the tide gauges are also to be seen in the Platzman synthesis of Fig. 2. Consequently, these findings can be taken as confirmation of the Platzman-Cartwright suggestion for the generation of M1. A possible exception concerns the pattern of amphidromes in the Pacific and the progression of phase lag along the American Pacific coast, which differ somewhat from Platzman's synthesis. However, this is probably acceptable given the low amplitudes in this region.

\section{Conclusions}

The method for determining M1' and M1 amplitudes and phases via Eqs. (2a) and (2b), and the availability of data from the large number of tide gauge records in the GESLA-2 data set, has enabled these small constituents to be studied on a near-global basis for the first time. Even though M1' and M1 may have small amplitudes, and at first sight it seems remarkable that they can be measured reliably at all, the general consistency of findings between neighbouring stations provides confidence in the results.

The worldwide distribution of M1' has been shown to be consistent with expectations for a degree- 2 diurnal tide, being similar in its general character to other diurnals (K1 and O1). As a result, Doodson's method of combination of four degree-2 terms into an overall M1' seems justified. However, there is some uncertainty regarding this way of computing M1' at stations around the UK and on the NW European continental coastline where the interaction between $\mathrm{N} 2$ and $\mathrm{O} 1$ into NO1, at the same frequency as line (7), could provide a complication. It seems that to do a better job for M1' around the UK, one must abandon the Doodson assumption of nodal factors in Eq. (1) and return to the analysis of long, nearcomplete records of (usually) hourly data, as Amin (1982) demonstrated.

A much better situation applies for M1, with excellent agreement between the results of methods used in previous and present studies (Tables 2 and 3). This is the first time that this esoteric degree- 3 ocean tide has been studied in any detail beyond NW Europe, where it was first identified, and the coastlines of the Atlantic Ocean. Inspection of tide gauge findings in Fig. 7a and $b$ and of the spatial variations in M1 amplitude and phase from the numerical model in Fig. 8a, b, indicates that the Platzman-Cartwright suggestion was correct. The ocean responds to degree- 3 forcing at M1 frequency as a result of normal modes with similar frequencies, consistent with Cartwright's findings from the Atlantic and also now from the other ocean basins. In particular, the small amplitudes over most of the central Pacific and Indian oceans, the larger amplitudes along the NE Pacific coast, and the apparent rotation of phase lag in the Indian Ocean are all qualitatively in agreement with the Platzman synthesis in Fig. 2.

However, there are also detailed differences on a regional scale, for example the different phases observed in the tide gauge data either side of New Zealand and Japan, which remain for further study. And on a local scale, there are anomalies in estuaries and inlets where shallow-water processes might result in apparent M1 generation, although it is not immediately clear how (interaction of $\mathrm{N} 2$ and $\mathrm{O} 1$, for example, would generate an apparent M1' rather than M1). Such non-linear processes for M1 generation on a larger scale were considered and rejected by Cartwright (1975).

Consequently, while the maps of Fig. S7a, b and in the Supplement are interesting, and their qualitative agreement with the numerical model and with Platzman's synthesis is 
reassuring, there is clearly a need for more detailed studies using more advanced ocean tide modelling. To our knowledge, a global model for M1 has never been constructed until now. For example, M1 is not included in the Finite Element Solution 2014 model (FES, 2018) that is now used by many groups. Such studies would provide further insight into how the ocean responds to a different (degree-3) form of tidal forcing and would be an important extension of the pioneering work on normal modes of the world ocean by Platzman.

Data availability. All tide gauge data used in this paper may be obtained from https://www.gesla.org (last access: 16 April 2019). Full details of the GESLA-2 data set are given by Woodworth et al.(2017; https://doi.org/10.1002/gdj3.42).

Supplement. The supplement related to this article is available online at: https://doi.org/10.5194/os-15-431-2019-supplement.

Competing interests. The author declares that there is no conflict of interest.

Special issue statement. This article is part of the special issue "Developments in the science and history of tides (OS/ACP/HGSS/NPG/SE inter-journal SI)". It is not associated with a conference.

Acknowledgements. I would not have had the idea for this paper without the previous work by David Cartwright and colleagues at Bidston Observatory. I am grateful to Cartwright in particular, to Roger Flather for the model code, and to David Pugh, Ian Vassie, Richard Ray and others for discussions about tides. Richard Ray is also thanked for sharing his analyses of UK records. Some figures in this paper were generated using the Generic Mapping Tools (Wessel and Smith, 1998). Jo Williams, Ivan Haigh and an anonymous reviewer provided valuable comments.

Review statement. This paper was edited by Mattias Green and reviewed by Ivan Haigh and one anonymous referee.

\section{References}

Agnew, D. C.: Earth Tides, in: Treatise on Geophysics, Volume 3 (Geodesy), edited by: Herring, T. A., Elsevier, New York, ISBN 9780444534606. 163-195, 2007.

Amin, M.: On analysis and prediction of tides on the west coast of Great Britain, Geophys. J. Roy. Astr. S., 68, 57-78, https://doi.org/10.1111/j.1365-246X.1982.tb06962.x, 1982.

Amin, M.: Temporal variations of tides on the west coast of Great Britain, Geophys. J. Roy. Astr. S., 82, 279-299, https://doi.org/10.1111/j.1365-246X.1985.tb05138.x, 1985.
Bell, C., Vassie, J. M., and Woodworth, P. L.: The Tidal Analysis Software Kit (TASK-2000 Package), National Oceanography Centre, Liverpool, 1998.

Cartwright, D. E.: A subharmonic lunar tide in the seas off Western Europe, Nature, 257, 277-280, https://doi.org/10.1038/257277a0, 1975.

Cartwright, D. E.: Anomalous M1 tide at Lagos, Nature, 263, $217-$ 218, https://doi.org/10.1038/263217b0, 1976.

Cartwright, D. E. and Tayler, R. J.: New computations of the tide-generating potential, Geophys. J. Roy. Astr. S., 23, 45-74, https://doi.org/10.1111/j.1365-246X.1971.tb01803.x, 1971.

Cartwright, D. E. and Edden, A. C.: Corrected tables of tidal harmonics, Geophys. J. Roy. Astr. S., 33, 253-264, https://doi.org/10.1111/j.1365-246X.1973.tb03420.x, 1973.

Cartwright, D. E., Spencer, R., Vassie, J. M., and Woodworth, P. L.: The tides of the Atlantic Ocean, $60 \mathrm{~N}$ to $30 \mathrm{~S}$, Philos. T. R. Soc. S-A, 324, 513-563, https://doi.org/10.1098/rsta.1988.0037, 1988.

Doodson, A. T.: The harmonic development of the tidegenerating potential, Philos. T. R. Soc. S-A, 100, 305-329, https://doi.org/10.1098/rspa.1921.0088, 1921.

Doodson, A. T.: VI. The analysis of tidal observations, Philos. T. R. Soc. S-A, 227, 223-279, https://doi.org/10.1098/rsta.1928.0006, 1928.

Doodson, A. T. and Warburg, H. D.: Admiralty Manual of Tides, His Majesty's Stationery Office, 270 pp., 1941.

FES: Description of the FES2014 ocean tide model, available at: https://www.aviso.altimetry.fr/en/data/products/ auxiliary-products/global-tide-fes/description-fes2014.html, last access: 1 October 2018.

Flather, R. A.: A numerical model investigation of tides and diurnal-period continental shelf waves along Vancouver Island, J. Phys. Oceanogr., 18, 115-139, https://doi.org/10.1175/15200485(1988)018<0115:ANMIOT>2.0.CO;2, 1988.

Foreman, M. G. G., Crawford, W. R., Cherniawsky, J. Y., Henry, R. F., and Tarbotton, M. R.: A high-resolution assimilating tidal model for the northeast Pacific Ocean, J. Geophys. Res., 105, 28629-28651, https://doi.org/10.1029/1999JC000122, 2000.

International Hydrographic Organization (IHO): Harmonic constants product specification. Edition 1.0. Tides Committee of the International Hydrographic Organization, Dated November 2006, 29 pp., 2006.

Mosetti, F. and Manca, B.: Some methods of tidal analysis, Int. Hydrogr. Rev., 59, 107-120, 1972.

Pingree, R. D. and Griffiths, D. K.: Tidal friction and the diurnal tides on the North-West European Shelf, J. Mar. Biol. Assoc. UK, 62, 577-593, https://doi.org/10.1017/S0025315400019767, 1982.

Platzman, G. W.: Normal modes of the world ocean. Part III: a procedure for tidal synthesis, J. Phys. Oceanogr., 14, 1521-1531, https://doi.org/10.1175/15200485(1984)014<1521:NMOTWO>2.0.CO;2, 1984a.

Platzman, G. W.: Normal modes of the world ocean. Part IV: synthesis of diurnal and semidiurnal tides, J. Phys. Oceanogr., 14, 1532-1550, https://doi.org/10.1175/15200485(1984)014<1532:NMOTWO>2.0.CO;2, 1984b.

Pugh, D. T. and Woodworth, P. L.: Sea-level science: Understanding tides, surges, tsunamis and mean sea-level changes, Cambridge 
University Press, Cambridge, ISBN 9781107028197, 408 pp., 2014.

Ray, R. D.: Resonant third-degree diurnal tides in the seas off Western Europe, J. Phys. Oceanogr., 31, 3581-3586, https://doi.org/10.1175/15200485(2001)031<3581:RTDDTI>2.0.CO;2, 2001.

Ray, R. D.: Secular changes in the solar semidiurnal tide of the western North Atlantic Ocean, Geophys. Res. Lett., 36, L19601, https://doi.org/10.1029/2009GL040217, 2009.

Ross, A. C., Najjar, R. G., Li, M., Lee, S. B., Zhang, F., and Liu, W.: Fingerprints of sea level rise on changing tides in the Chesapeake and Delaware Bays, J. Geophys. Res.-Oceans, 122, 8102-8125, https://doi.org/10.1002/2017JC012887, 2017.

Stammer, D., Ray, R. D., Andersen, O. B., Arbic, B. K., Bosch, W., Carrère, L., Cheng, Y., Chinn, D. S., Dushaw, B. D., Egbert, G. D., Erofeeva, S. Y., Fok, H. S., Green, J. A. M., Griffiths, S., King, M. A., Lapin, V., Lemoine, F. G., Luthcke, S. B., Lyard, F., Morison, J., Müller, M., Padman, L., Richman, J. G., Shriver, J. F., Shum, C. K., Taguchi, E., and Yi, Y.: Accuracy assessment of global barotropic ocean tide models, Rev. Geophys., 52, $243-$ 282, https://doi.org/10.1002/2014RG000450, 2014.

Voinov, G. N.: Anomalous diurnal tides of the third degree in the Barents and Kara Seas, Dokl. Earth Sci., 438, 693-696, https://doi.org/10.1134/S1028334X11050278, 2011.
Wahr, J. M.: Body tides on an elliptical, rotating, elastic and oceanless Earth, Geophys. J. Roy. Astr. S., 64, 677-703, https://doi.org/10.1111/j.1365-246X.1981.tb02690.x, 1981.

Weatherall, P., Marks, K. M., Jakobsson, M., Schmitt, T., Tani, S., Arndt, J. E., Rovere, M., Chayes, D., Ferrini, V., and Wigley, R.: A new digital bathymetric model of the world's oceans, Earth Space Sci., 2, 331-345, https://doi.org/10.1002/2015EA000107, 2015.

Wessel, P. and Smith, W. H. F.: New, improved version of generic mapping tools released, EOS T. Am. Geophys. Un., 79, p. 579, 1998.

Woodworth, P. L., Hunter, J. R. Marcos, M., Caldwell, P., Menéndez, M., and Haigh, I.: Towards a global higherfrequency sea level data set, Geosci. Data J., 3, 50-59, https://doi.org/10.1002/gdj3.42, 2017.

Woodworth, P. L. and Hibbert, A.: The nodal dependence of longperiod ocean tides in the Drake Passage, Ocean Sci., 14, 711-730, https://doi.org/10.5194/os-14-711-2018, 2018. 